Research Paper

\title{
Clinical value of docetaxel plus cisplatin (TP) induction chemotherapy followed by TP concurrent chemoradiotherapy in locoregionally advanced nasopharyngeal carcinoma
}

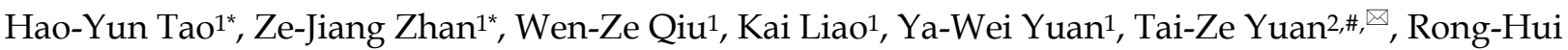 \\ Zheng1,\#, 凶।
}

1. Department of Radiation Oncology, Affiliated Cancer Hospital \& Institute of Guangzhou Medical University, Guangzhou, 510090, Guangdong, P. R. China.

2. Department of Radiation Oncology, Guangzhou Concord Cancer Center, Guangzhou, 510045, Guangdong, P. R. China.

* Contributed equally as co-first authors.

\#Contributed equally as correspondence authors

$\square$ Corresponding authors: Rong-Hui Zheng and Tai-Ze Yuan, Department of Radiation Oncology, Affiliated Cancer Hospital \& Institute of Guangzhou Medical University, Guangzhou 510095, Guangdong, P. R. China and Department of Radiation Oncology, Guangzhou Concord Cancer Center, Guangzhou 510045, Guangdong, P. R. China, respectively. Tel: +86-02066673666 and +86-02022050822, respectively. E-mail addresses: zhengronghui@gzhmu.edu.cn (Rong-Hui Zheng), taize.yuan@ccm.cn (Tai-Ze Yuan).

() The author(s). This is an open access article distributed under the terms of the Creative Commons Attribution License (https://creativecommons.org/licenses/by/4.0/). See http://ivyspring.com/terms for full terms and conditions.

Received: 2020.06.25; Accepted: 2020.10.25; Published: 2021.01.01

\begin{abstract}
Objective: To investigate the clinical value of induction chemotherapy (IC) with docetaxel plus cisplatin (TP) followed by concurrent chemoradiotherapy (CCRT) with TP in locoregionally advanced nasopharyngeal carcinoma (NPC).

Methods: A total of 544 patients with locoregionally advanced NPC that was newly diagnosed from January 2009 to December 2015 were included in this study. Among these patients, 251 were treated with TP induction chemotherapy followed by CCRT with cisplatin (DDP) alone (TP + DDP group), 167 were treated with TP followed by CCRT with TP (TP + TP group), and 126 were treated with docetaxel, DDP and fluorouracil (TPF) followed by CCRT with DDP alone (TPF + DDP group). Overall survival (OS), distant metastasis-free survival (DMFS), progression-free survival (PFS) and locoregional relapse-free survival (LRRFS) were analyzed using the Kaplan-Meier method and a Cox proportional hazards model.

Results: Survival analysis showed that the 5-year OS, PFS and DMFS rates in the TP + DDP group were significantly lower than those in the TP + TP group after propensity score matching (PSM). Multivariate analysis revealed that CCRT with TP was an independent prognostic factor for OS, PFS and DMFS. During CCRT, the incidence rates of grade $3 / 4$ nausea/vomiting, oral mucositis, leukocytopenia and neutropenia were significantly increased in the TP + TP group compared with the TP + DDP group (all $P$ $<0.05)$. To further explore the value of TP + TP, we performed PSM again with the TPF + DDP group. After PSM, there were 100 patients in each group. Survival analysis showed no significant differences in the 5-year OS, PFS, DMFS and LRRFS rates between the two groups. During IC and CCRT, the rate of grade $3 / 4$ nausea/vomiting in the TPF + DDP group was higher than that in the TP+TP group $(9.0 \%$ vs. $2.0 \%, P=0.030 ; 18.0 \%$ vs. $8.0 \%, P=0.036$, respectively). No significant difference in the incidence of grade $3 / 4$ hematologic toxicity was found between the two groups (all $P>0.05$ ).

Conclusion: TP + TP can reduce the distant metastasis of locoregionally advanced NPC and improve OS compared with TP + DDP; TP + TP has the same effect as TPF + DDP and is clinically feasible.
\end{abstract}

Key words: Nasopharyngeal carcinoma, concurrent chemoradiotherapy, propensity score matching, docetaxel, cisplatin 


\section{Introduction}

Nasopharyngeal carcinoma (NPC), originating from nasopharyngeal mucosa epithelia, is a common malignant tumor of the head and neck. Patients suffering from NPC are mainly concentrated in southeast Asia, especially in South China, and there are obvious regional characteristics[1]. The morbidity of NPC is 20 30 per 100,000 people in Guangdong Province[2]. Because of a lack of specificity of the early symptoms, $70 \%$ of patients diagnosed with NPC are in advanced stages[3,4]. Radiotherapy is the main treatment for NPC because the primary tumor exhibits a unique anatomical location and radiosensitivity[1,5].

The intergroup 0099 trial demonstrated that concurrent chemoradiotherapy (CCRT) with high-dose cisplatin (DDP) followed by adjuvant chemotherapy (AC) significantly improved overall survival (OS) and progression-free survival (PFS) in patients with locoregionally advanced NPC, which was confirmed by several large clinical studies in an NPC endemic area[6-9]. Therefore, CCRT with DDP followed by $\mathrm{AC}$ has become the standard treatment. However, a phase III clinical trial found that the addition of AC followed by CCRT could not improve the 5-year OS and PFS rates compared with CCRT alone $(80 \%$ vs. $83 \%, P=0.35 ; 71 \%$ vs. $75 \%, P=0.45$, respectively) in patients with locoregionally advanced NPC[10]. These negative results may be attributed to the fact that only $63 \%$ of patients could complete the entire course of AC. Thus, whether AC can further improve patient survival is still unknown.

Induction chemotherapy (IC) has the advantages of better tolerance and early removal of micrometastases[11]. Sun et al.[12] reported that docetaxel, cisplatin and fluorouracil (TPF) followed by CCRT with DDP alone (TPF + DDP) significantly improved OS, PFS and distant metastasis-free survival (DMFS) in patients with locoregionally advanced NPC. An individual patient data (IPD) pooled analysis recruiting four clinical trials demonstrated the positive clinical value of IC[13]. Therefore, the 2018 National Comprehensive Cancer Network (NCCN) upgraded the evidence of IC to category 2A[14]. However, Hui et al.[15] found that compared with CCRT alone, docetaxel and cisplatin (TP) followed by CCRT with DDP alone (TP + DDP) could not improve the 3 -year PFS (hazard ratio $(\mathrm{HR})=$ $0.49 ; 95 \%$ confidence interval (CI) $0.20-1.19 ; P=0.12$ ) in locoregionally advanced NPC. Therefore, further improvement of the TP + DDP regimen is needed.

Furthermore, the role of CCRT with a double-drug regimen in locoregionally advanced NPC is unknown. CCRT with a double-drug regimen in lung cancer and cervical cancer is approved and widely used in clinical practice[16, 17]. Radiotherapy combined with docetaxel is feasible and is widely used in non-small-cell lung cancer (NSCLC) and cervical cancer[18, 19]. Recently, a study demonstrated that CCRT combined with a small weekly dose of docetaxel is an effective and tolerable treatment for locoregionally advanced NPC[20]. It is not clear whether CCRT with a double-drug (docetaxel + DDP) regimen could improve survival compared with CCRT with DDP alone.

Therefore, this study aimed to explore the significance of TP-based IC followed by CCRT with a $\mathrm{TP}$ regimen in locoregionally advanced NPC.

\section{Patient selection and methods}

\section{Patient selection}

This study was approved by the ethics committee of the Affiliated Cancer Hospital \& Institute of Guangzhou Medical University. Clinical and imaging data were collected from 1218 patients with newly diagnosed locoregionally advanced NPC who received IC followed by CCRT (IC + CCRT) at the Department of Radiation Oncology of the Affiliated Cancer Hospital \& Institute of Guangzhou Medical University from January 2009 to December 2015. The inclusion criteria were as follows: (1) patients with a pathologic diagnosis of WHO type I, II and III NPC; (2) patients who underwent definitive intensity-modulated radiotherapy (IMRT); (3) patients with a performance status (PS) score between 0 and 1 ; (4) patients with stage III-IVa disease according to the $8^{\text {th }}$ edition of the Union for International Cancer Control/American Joint Committee on Cancer (UICC/AJCC) staging system; (5) patients administered CCRT with DDP or TP after TP induction chemotherapy; (6) patients who received TPF induction chemotherapy followed by CCRT with DDP alone; and (7) patients who received no adjuvant chemotherapy. The exclusion criteria were as follows: (1) previous history of other malignancies; (2) evidence of serious dysfunction of heart, lung, liver, kidney and other important organs; and (3) patients who were pregnant or nursing. After screening, 674 patients were excluded, of whom 150 were initially diagnosed with stage IVb or I/II patients, 248 were not treated with TPF or TP induction chemotherapy regimen or DDP-based CCRT, and 276 were treated with AC. Finally, 544 patients were included in this study, and patients were classified into three treatment groups: patients in the TP + DDP group were treated with TP induction chemotherapy followed by CCRT with DDP alone $(\mathrm{n}=251)$; patients in the TP + TP group were treated with TP induction 
chemotherapy followed by CCRT with TP ( $\mathrm{n}=167)$; and patients in the TPF + DDP group were treated with TPF-based IC followed by CCRT with DDP alone $(\mathrm{n}=126)$. The details are shown in Figure 1.

\section{Induction chemotherapy}

An IC regimen with TPF or TP was used in this study. The TPF regimen included docetaxel (60 $\left.\mathrm{mg} / \mathrm{m}^{2}, \mathrm{~d} 1\right)$, DDP $\left(60 \mathrm{mg} / \mathrm{m}^{2}, \mathrm{~d} 1\right)$ and fluorouracil $\left(600 \mathrm{mg} / \mathrm{m}^{2}, 24\right.$ hours daily from $\left.\mathrm{d} 1 \sim 5\right)$. The TP regimen included docetaxel $\left(75 \sim 80 \mathrm{mg} / \mathrm{m}^{2}, \mathrm{~d} 1\right)$ and DDP $\left(80 \mathrm{mg} / \mathrm{m}^{2}, \mathrm{~d} 1\right)$. IC was administered every three weeks.

\section{Concurrent chemoradiotherapy}

Radiotherapy is the main treatment for NPC. All patients were irradiated with IMRT. After being immobilized with head and neck thermoplastic masks while in the supine position, patients were scanned with a CT simulator. Noncontrast and contrast CT images were collected with $3 \mathrm{~mm}$ per slice from the vertex to $2 \mathrm{~cm}$ below the clavicle head. The target volumes were contoured according to the Sun Yat-sen University Cancer Center institutional treatment protocol[21], which is in agreement with the International Commission on Radiation Units and Measurements Reports 50 and 62. The prescribed radiation dose to the gross tumor volume of nasopharyngeal tumors (GTVnx) was 70 74 Gy, and the prescribed radiation dose to the gross tumor volume of lymph nodes (GTVnd) was 68 70 Gy. The high-risk clinical target volume (CTV1) and the low-risk clinical target volume (CTV2) were prescribed 60 66 Gy and 54 56 Gy, respectively. All patients were irradiated with 30 to 32 fractions in total, once daily, Monday through Friday. Patients in the TP + DDP group or the TPF + DDP group were treated with 1 3 cycles of DDP $\left(80 \sim 100 \mathrm{mg} / \mathrm{m}^{2}\right.$, d1) every 21 days during radiotherapy. Patients in the TP + TP group were given DDP $\left(75 \sim 80 \mathrm{mg} / \mathrm{m}^{2}, \mathrm{~d} 1\right)$ and docetaxel $\left(75 \mathrm{mg} / \mathrm{m}^{2}, \mathrm{~d} 1\right)$ treatment, which was repeated every 21 days for $1 \sim 3$ cycles during radiotherapy.

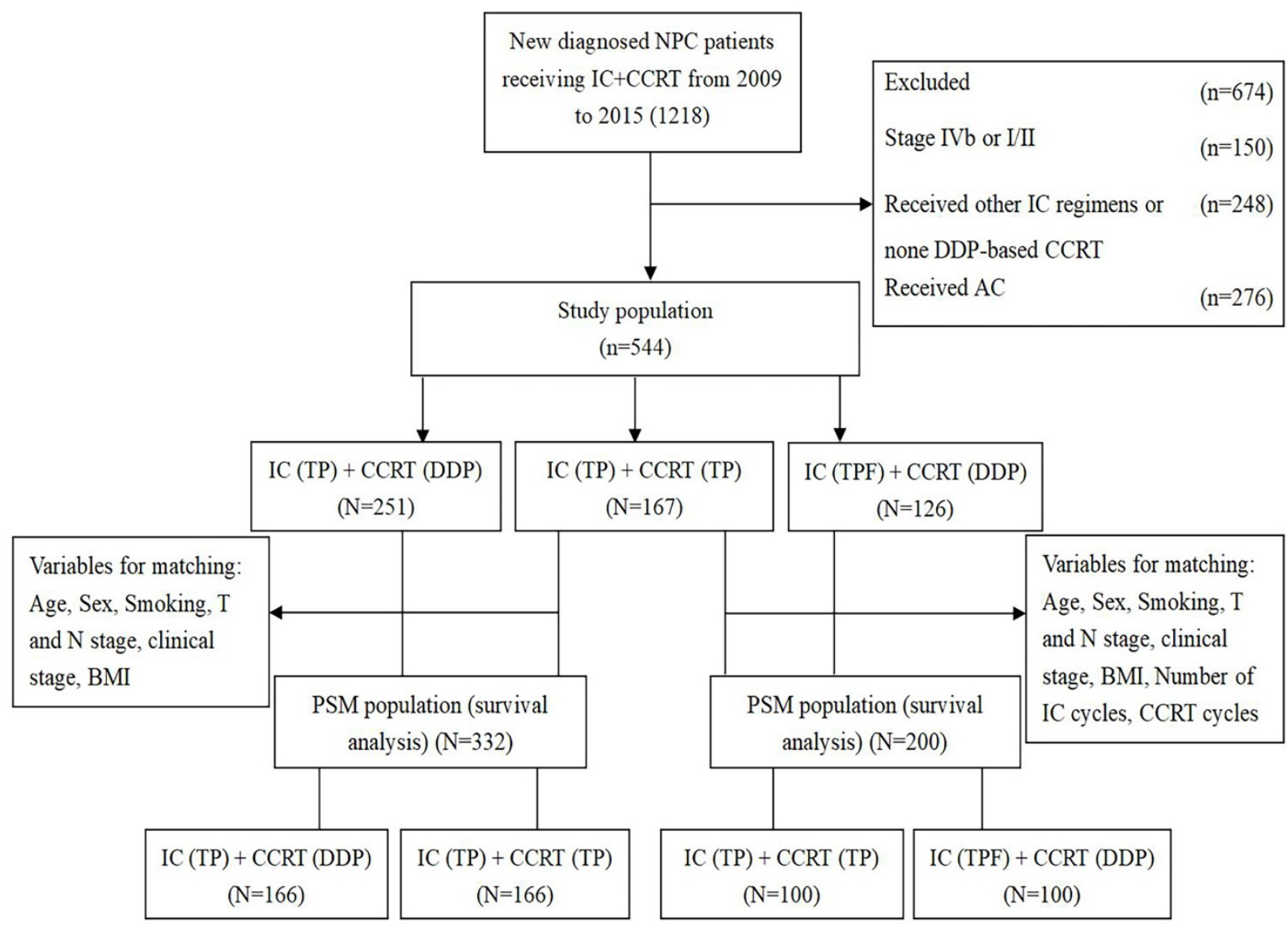

Fig. 1. Flow chart of patient selection. IC: induction chemotherapy; CCRT: concurrent chemoradiotherapy; TP: docetaxel and cisplatin; TPF: docetaxel, cisplatin and fluorouracil; PSM: propensity score matching; DDP: cisplatin; BMI: body mass index. 


\section{Follow-up}

After treatment, the patients were followed every 3 months for 2 years, every 6 months from the $3^{\text {rd }}$ to $5^{\text {th }}$ years, and every year thereafter. Follow-up data were obtained from outpatient and inpatient medical records and telephone counseling. The follow-up time was calculated from the date of diagnosis to the last date of follow-up or death. OS was calculated from the date of diagnosis until the follow-up deadline or death. PFS was defined as the time from initial diagnosis to tumor progression or death; DMFS was defined as the time to tumor metastasis; and locoregional relapse-free survival (LRRFS) was defined as the time to the first locoregional recurrence. Hematologic toxicity, liver and kidney function, and oral mucosal responses were graded according to the National Cancer Institute Common Terminology Criteria for Adverse Events Version 4.0 (NCI-CTCAE 4.0)[22].

\section{Data analysis}

SPSS 25.0 (Chicago, IL, USA) was used for all data analysis. Clinical features and toxicities were analyzed using the chi-square test or Fisher's test. In this study, propensity score matching (PSM) was used to exclude observable confounding factors[23], and covariates were included in the PSM analysis, including age, sex, smoking history, T stage, $\mathrm{N}$ stage, clinical stage, pretreatment body mass index (BMI), and number of IC and CCRT cycles. The survival outcomes of the two groups' PSM cohort were analyzed by the Kaplan-Meier method. Multivariate analysis was performed using a Cox proportional hazards regression model, and the analyzed variables included T stage, $\mathrm{N}$ stage, clinical stage, age, smoking history, and number of IC courses, CCRT cycles and CCRT regimens. All statistical analyses were defined with significance level of $P<0.05$. All trials were bilateral, and the results of the multivariate analysis were expressed by both the HR and the 95\% CI.

\section{Results}

Before PSM, there were 418 patients in total, with 251 patients in the TP + DDP group and 167 patients in the TP + TP group. After PSM, 332 patients were included, with 166 patients in each group. The baseline clinical characteristics of the TP + DDP and $\mathrm{TP}+\mathrm{TP}$ groups are shown in Table 1 .

The median follow-up time of 332 patients was 64 months (4 114 months), and the 5-year OS, PFS, DMFS and LRRFS rates were $84.9 \%, 79.4 \%, 85.8 \%$ and $90.6 \%$, respectively. The 5-year OS, PFS, DMFS and LRRFS rates in the TP + DDP group vs. TP + TP group were $81.5 \%$ vs. $88.8 \%, 74.7 \%$ vs. $84.5 \%, 82.0 \%$ vs. $89.8 \%$, and $91.8 \%$ vs. $89.6 \%$, respectively $(P=0.040$,
Fig. 2A; $P=0.024$, Fig. 2B; $P=0.029$, Fig. $2 \mathrm{C}$; and $P=$ 0.667 , Fig. 2D, respectively).

Table 1. Characteristics of patients in the TP + TP and TP + DDP groups before and after propensity score matching

\begin{tabular}{|c|c|c|c|c|c|c|}
\hline Item & Entire col & hort (\%) & & Propensity & e-matched & $t(\%)$ \\
\hline & TP+DDP & $\mathrm{TP}+\mathrm{TP}$ & $P$ & TP+DDP & $\mathrm{TP}+\mathrm{TP}$ & $P$ \\
\hline Total & $\begin{array}{l}251 \\
(60.0)\end{array}$ & $\begin{array}{l}167 \\
(40.0)\end{array}$ & & $166(50.0)$ & $166(50.0)$ & \\
\hline Age (y) & & & 0.765 & & & 0.509 \\
\hline$<46$ & $\begin{array}{l}118 \\
(47.0)\end{array}$ & $81(48.5)$ & & 74 (44.6) & $80(48.2)$ & \\
\hline$\geq 46$ & $\begin{array}{l}133 \\
(53.0)\end{array}$ & $86(51.5)$ & & $92(55.4)$ & $86(51.8)$ & \\
\hline Gender & & & 0.672 & & & 0.536 \\
\hline Male & $\begin{array}{l}174 \\
(69.3)\end{array}$ & $\begin{array}{l}119 \\
(71.3)\end{array}$ & & 124 (74.7) & 119 (71.7) & \\
\hline Female & $77(30.7)$ & $48(28.7)$ & & $42(25.3)$ & $47(28.3)$ & \\
\hline T stage & & & $\begin{array}{l}< \\
0.001\end{array}$ & & & 0.110 \\
\hline T1-2 & $41(16.3)$ & $53(31.7)$ & & $39(23.5)$ & $52(31.3)$ & \\
\hline T3-4 & $\begin{array}{l}210 \\
(83.7)\end{array}$ & $\begin{array}{l}114 \\
(68.3)\end{array}$ & & 127 (76.5) & 114 (68.7) & \\
\hline N stage & & & 0.738 & & & 1.000 \\
\hline N0-1 & $79(31.5)$ & $54(32.3)$ & & $54(32.5)$ & $54(32.5)$ & \\
\hline $\mathrm{N} 2-3$ & $\begin{array}{l}172 \\
(68.5)\end{array}$ & $\begin{array}{l}113 \\
(67.7)\end{array}$ & & $112(67.5)$ & $112(68.5)$ & \\
\hline $\begin{array}{l}\text { Clinical } \\
\text { stage }\end{array}$ & & & 0.449 & & & 0.720 \\
\hline III & $\begin{array}{l}167 \\
(66.5)\end{array}$ & $\begin{array}{l}117 \\
(70.1)\end{array}$ & & 114 (68.7) & 117 (70.5) & \\
\hline IVa & $84(33.5)$ & $50(29.9)$ & & $52(31.3)$ & $49(29.5)$ & \\
\hline Smoking & & & 0.673 & & & 0.659 \\
\hline Yes & $\begin{array}{l}103 \\
(41.0)\end{array}$ & $72(36.5)$ & & $76(45.8)$ & $72(43.4)$ & \\
\hline No & $\begin{array}{l}148 \\
(59.0)\end{array}$ & $95(56.9)$ & & $90(54.2)$ & 94 (56.6) & \\
\hline CCRT cycles & & & 0.166 & & & 0.667 \\
\hline$<2$ & $56(22.3)$ & $28(16.8)$ & & $31(18.7)$ & $28(16.9)$ & \\
\hline$\geq 2$ & $\begin{array}{l}195 \\
(77.7)\end{array}$ & $\begin{array}{l}139 \\
(83.2)\end{array}$ & & 135 (81.3) & $138(83.1)$ & \\
\hline IC cycles & & & 0.721 & & & 0.158 \\
\hline$<2$ & $63(25.1)$ & $47(28.1)$ & & $59(35.5)$ & $47(28.3)$ & \\
\hline$\geq 2$ & $\begin{array}{l}188 \\
(74.9)\end{array}$ & $\begin{array}{l}120 \\
(71.9)\end{array}$ & & $107(64.5)$ & 119 (71.7) & \\
\hline BMI $\left(\mathrm{kg} / \mathrm{m}^{2}\right.$ & & & 0.977 & & & 0.859 \\
\hline$<18$ & $11(4.4)$ & $8(4.8)$ & & $6(3.6)$ & $8(4.8)$ & \\
\hline $18-24$ & $\begin{array}{l}164 \\
(65.3)\end{array}$ & $\begin{array}{l}108 \\
(64.7)\end{array}$ & & 109 (65.7) & $107(64.5)$ & \\
\hline$>24$ & $76(30.3)$ & $51(30.5)$ & & $51(30.7)$ & $51(30.7)$ & \\
\hline Histology & & & 0.882 & & & 0.598 \\
\hline I & $1(0.4)$ & $1(0.6)$ & & $1(0.6)$ & $1(0.6)$ & \\
\hline II & $6(2.4)$ & $3(1.8)$ & & $6(3.6)$ & $3(1.8)$ & \\
\hline III & $\begin{array}{l}244 \\
(97.2)\end{array}$ & $\begin{array}{l}163 \\
(97.6)\end{array}$ & & 159 (95.8) & $162(97.6)$ & \\
\hline
\end{tabular}

IC: induction chemotherapy; CCRT: concurrent chemoradiotherapy; TP: docetaxel and cisplatin; DDP: cisplatin; BMI: body mass index.

As shown in Table 2, Cox regression multivariate analysis was used to adjust for various prognostic factors. Grouping factors of $\mathrm{TP}+\mathrm{TP}$ significantly improved the 5-year OS (HR, 0.563; 95\% CI 0.325 to $0.974 ; P=0.048)$, PFS (HR, $0.585 ; 95 \%$ CI 0.359 to 0.953 ; $P=0.031$ ) and DMFS (HR, 0.523; $95 \%$ CI 0.285 to 0.961 ; $P=0.037)$. Clinical staging was an independent prognostic factor for OS, PFS, DMFS and LRRFS (all $P$ $<0.05)$. $\mathrm{N}$ staging and IC cycles was an independent prognostic factor for OS, PFS and DMFS (all $P<0.05$ ). 
Table 2. Multivariate analysis of prognostic factors in 332 patients with NPC after propensity score matching

\begin{tabular}{lllll}
\hline Endpoint & Variable & Hazard ratio & $95 \%$ CI & $P$ \\
\hline OS & Group (TP+TP vs. & 0.563 & $(0.325-0.974)$ & 0.048 \\
& TPF+DDP) & & & \\
& N stage & 2.281 & $(1.189-4.378)$ & 0.013 \\
& Clinical stage (III vs. & 2.699 & $(1.589-4.585)$ & 0.000 \\
& IVa) & & & \\
& IC cycles (<2 cycles vs. & 0.544 & $(0.318-0.929)$ & 0.026 \\
& $\geq 2$ cycles) & & & \\
PFS & Group (TP+TP vs. & 0.585 & $(0.359-0.953)$ & 0.031 \\
& TPF+DDP) & & \\
& N stage & 2.297 & $(1.266-4.165)$ & 0.006 \\
& Clinical stage (III vs. & 2.418 & $(1.496-3.908)$ & 0.000 \\
& IVa) & & \\
IC cycles (<2 cycles vs. & 0.558 & $(0.344-0.905)$ & 0.018 \\
& $\geq 2$ cycles) & & & \\
Group (TP+TP vs. & 0.523 & $(0.285-0.961)$ & 0.037 \\
DMFS & TPF+DDP) & & \\
& N stage & 2.740 & $(1.271-5.908)$ & 0.010 \\
& Clinical stage (III vs. & 2.694 & $(1.494-4.855)$ & 0.001 \\
IVa) & & & \\
& IC cycles (<2 cycles vs. 0.418 & $(0.232-0.752)$ & 0.004 \\
& $\geq 2$ cycles) & & & \\
& Clinical stage (III vs. & 2.154 & $(1.051-4.417)$ & 0.036 \\
& IVa) & & & \\
\hline
\end{tabular}

IC: induction chemotherapy; CCRT: concurrent chemoradiotherapy; TP: docetaxel and cisplatin; DDP: cisplatin; HR: hazard ratio.

Before PSM, 293 patients were divided into two groups, with 126 patients in the TPF + DDP group and 167 patients in the TP + TP group. After PSM, 200 patients were identified, and there were 100 patients in each cohort. The specific baseline characteristics are shown in Table 3. The median follow-up time of these

A
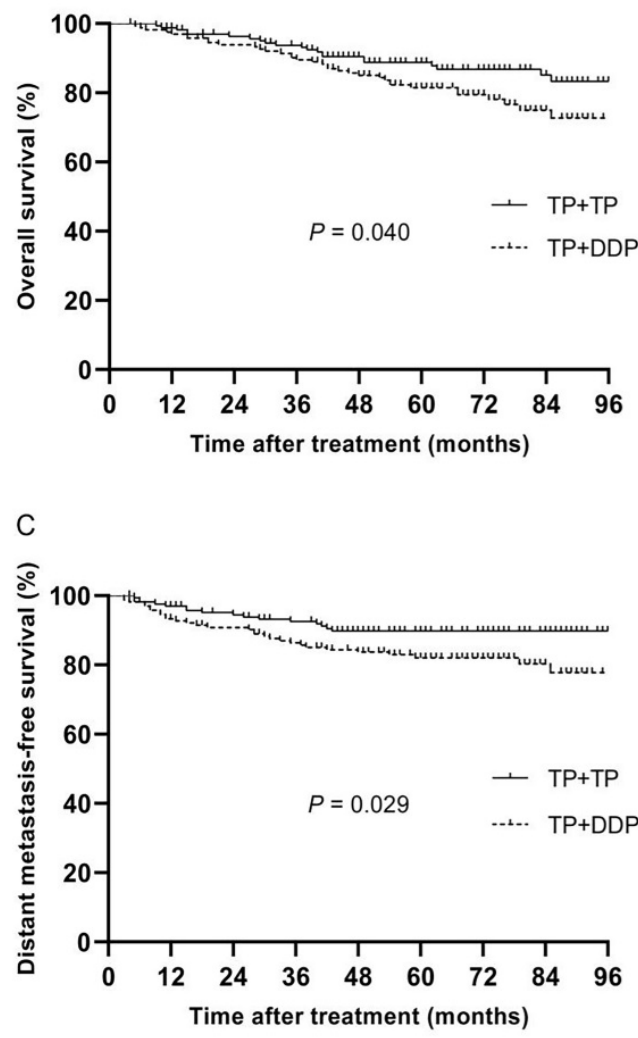

200 patients was 63 months (4 113 months), and the 5 -year OS, PFS, DMFS and LRRFS rates were $88.8 \%$, $83.5 \%, 89.9 \%$ and $91.6 \%$, respectively. The 5 -year OS, PFS, DMFS and LRRFS rates in the TPF + DDP group vs. TP + TP group were $88.0 \%$ vs. $89.7 \%, 84.4 \%$ vs. $82.9 \%, 89.5 \%$ vs. $90.5 \%$, and $92.6 \%$ vs. $91.0 \%$, respectively $(P=0.431$, Fig. $3 \mathrm{~A} ; P=0.801$, Fig. $3 \mathrm{~B} ; P=$ 0.893 , Fig. 3C; and $P=0.763$, Fig. 3D, respectively).

Multivariate analysis demonstrated that there were no significant differences between the TPF + DDP group and the TP + TP group in 5-year OS, PFS, DMFS and LRRFS. Clinical staging was an independent factor for OS, PFS and DMFS (Table 4).

\section{Toxic effects}

Table 5 shows patients' toxicities during IC in the PSM cohort. There were no differences in hematologic toxicities or nonhematologic toxicities between the TP + DDP group and the TP + TP group (all $P>0.05$ ). The hematologic toxicities in the TPF + DDP group were similar to those in the TP + TP group (all $P>0.05$ ). In terms of nonhematologic toxicities, the acute adverse events of grade $3 / 4$ nausea/vomiting in the TPF + DDP group were higher than those in the TP + TP group $(P=0.033)$. However, hypoalbuminemia, liver or kidney function did not show significant differences between the two groups (all $P>0.05$ ).

B
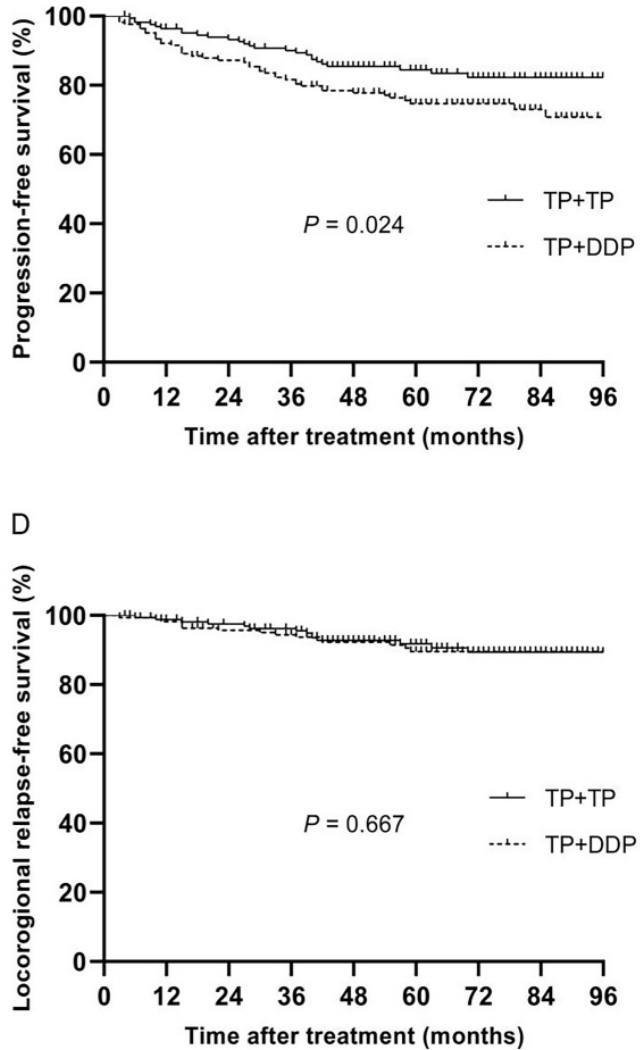

Fig. 2. Kaplan-Meier analysis of survival between TP + DDP and TP + TP groups after propensity score matching. A: Overall survival; B: Progression-free survival; C: Distant metastasis-free survival; D: Locoregional relapse-free survival. 
A

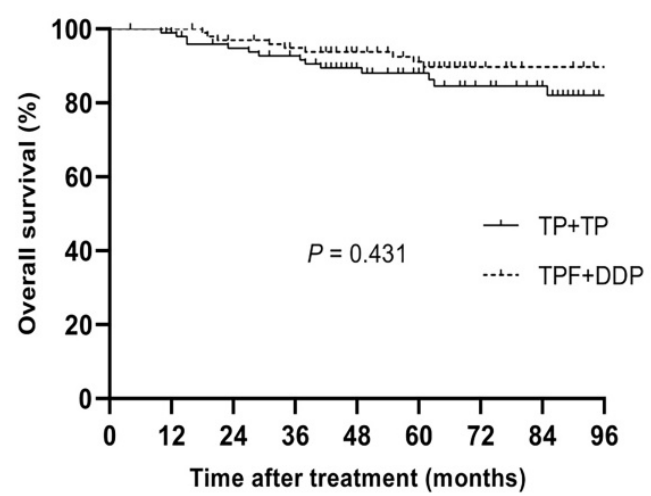

C

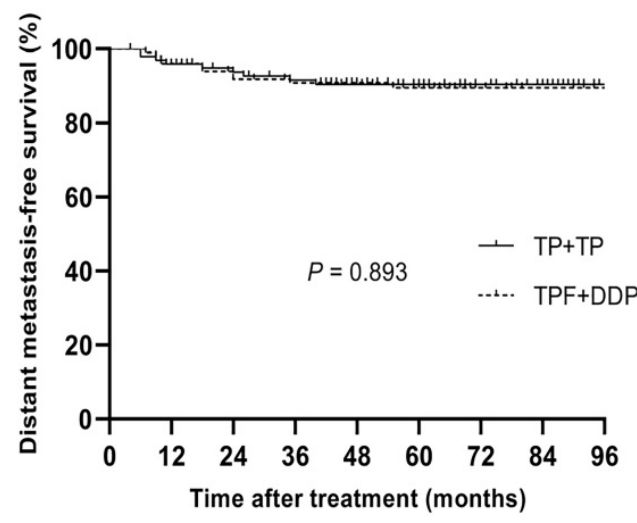

B

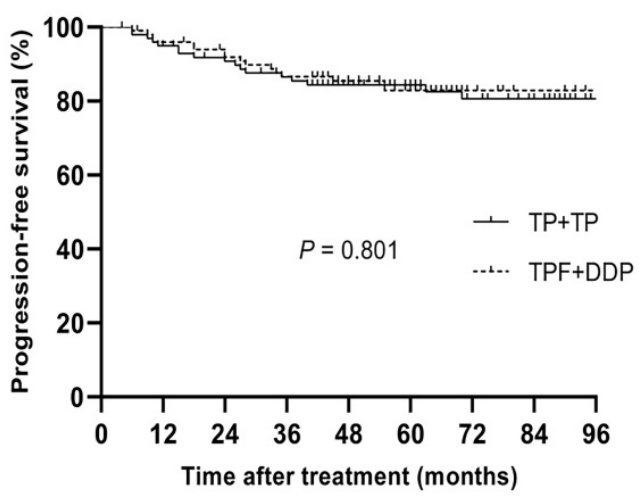

D

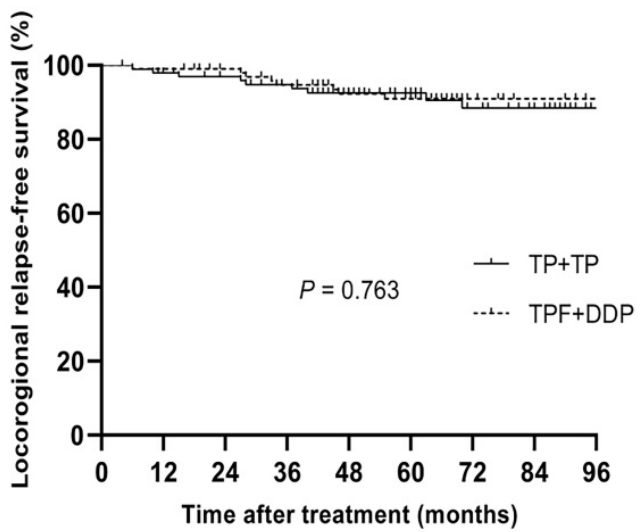

Fig. 3. Kaplan-Meier analysis of survival between TPF + DDP and TP + TP groups after propensity score matching. A: Overall survival; B: Progression-free survival; C: Distant metastasis-free survival; D: Locoregional relapse-free survival.

In the PSM cohort, patients' toxicities during CCRT are shown in Table 6 . The rates of grade $3 / 4$ leukocytopenia and neutropenia in the TP + TP group occurred in $44.0 \%$ and $39.8 \%$ of patients, respectively, which were significantly higher than those in the TP + DDP group (19.9\% and $16.3 \%$, respectively; $P<0.001)$. In terms of nonhematologic toxicities, the rates of grade $3 / 4$ oral mucosal and nausea/vomiting reactions in the $\mathrm{TP}+\mathrm{TP}$ group were significantly higher than those in the TP + DDP group $(P<0.001$ and $P=0.035$, respectively). The rates of acute adverse events of grade $3 / 4$ nausea/vomiting in the TPF + DDP group were higher than those in the TP + TP group $(18.0 \%$ vs. $8.0 \% ; P=0.036)$. However, there were no significant differences in other adverse events (all $P>0.05$ ).

\section{Discussion}

This study is the first to retrospectively explore the long-term efficacy and toxicities of CCRT with double-drug chemotherapy in locoregionally advanced NPC using PSM. First, under the condition of the same IC regimen, TP + TP group had superior
OS, PFS and DMFS over those in the TP + DDP group in locoregionally advanced NPC. Second, we found that the 5-year OS, PFS, DMFS and LRRFS rates were similar between the TP + TP group and the TPF + DDP group, but the rates of acute adverse events of grade $3 / 4$ nausea/vomiting responses were higher in the TPF + DDP group than in the $\mathrm{TP}+\mathrm{TP}$ group during the IC and CCRT period; this was the main clinical benefit in the TP + TP group.

Radiotherapy combined with DDP in locoregionally advanced NPC has been repeatedly demonstrated[7-9]. However, the distant metastasis rate still reached $30 \%$ in locoregionally advanced NPC[24]. Therefore, the addition of AC or IC has been used to decrease the distant metastasis rate in locoregionally advanced NPC. However, the significance of AC is still controversial in locoregionally advanced NPC[25]. Furthermore, compared with AC, IC can improve tolerance and eradicate micrometastases[11]. At present, several prospective studies have demonstrated the clinical efficacy of IC. Furthermore, an IPD pooled analysis including four clinical trials from endemic NPC regions confirmed that the IC + CCRT regimen could 
improve the 5 -year OS (HR $=0.75 ; 95 \%$ CI 0.90 to 0.51 ) and DMFS (HR $=0.68,95 \%$ CI 0.57 to 0.99)[13]. Hui et al.[15] showed the feasibility of IC with TP in locoregionally advanced NPC. However, the efficacy of the TP + DDP regimen was low, and the evidence level was only Category $2 \mathrm{~B}$ according to the NCCN guidelines[14]. Therefore, the TP + DDP regimen needs further investigation of the treatment of locally advanced NPC. Some studies demonstrated that the objective remission rate (ORR) of CCRT with the TP regimen was $100 \%$ in locoregionally advanced cervical cancer[26]. The RTOG 9410 reported that the 5 -year OS in stage III NSCLC with double-drug regimen CCRT was $16 \%$, and this has become the standard clinical treatment in stage III NSCLC[16]. The clinical effect of CCRT with a double-drug regimen in locoregionally advanced NPC is worth exploration.

Table 3. Characteristics of patients between the TPF + DDP group and TP+TP group before and after propensity score matching.

\begin{tabular}{|c|c|c|c|c|c|c|}
\hline Item & Entire coho & & & Propensity & matched col & \\
\hline & TPF+DDP & $\mathrm{TP}+\mathrm{TP}$ & $P$ & TPF+DDP & $\mathrm{TP}+\mathrm{TP}$ & $P$ \\
\hline Total & $126(43.0)$ & $167(57.0)$ & & $100(50.0)$ & $100(50.0)$ & \\
\hline Age & & & 0.905 & & & 0.887 \\
\hline$<46$ & $62(49.2)$ & $81(48.5)$ & & $51(51.0)$ & $52(52.0)$ & \\
\hline$\geq 46$ & $64(50.8)$ & $86(51.5)$ & & $49(49.0)$ & $48(48.0)$ & \\
\hline Gender & & & 0.114 & & & 0.626 \\
\hline Male & $100(79.4)$ & 119 (71.3) & & $76(76.0)$ & $73(73.0)$ & \\
\hline Female & $26(20.6)$ & $48(28.7)$ & & $24(24.0)$ & $27(27.0)$ & \\
\hline T stage & & & 0.181 & & & 1.000 \\
\hline T1-2 & 31 (24.6) & $53(31.7)$ & & $27(27.0)$ & $27(27.0)$ & \\
\hline T3-4 & 95 (75.4) & $114(68.3)$ & & $73(73.0)$ & $73(73.0)$ & \\
\hline N stage & & & 0.026 & & & 0.154 \\
\hline N0-1 & $26(20.6)$ & $54(32.3)$ & & $23(23.0)$ & $32(32.0)$ & \\
\hline N2-3 & $100(79.4)$ & $113(67.7)$ & & $77(77.0)$ & $68(68.0)$ & \\
\hline Clinical stage & & & 0.003 & & & 0.884 \\
\hline III & $67(53.2)$ & $117(70.1)$ & & $62(62.0)$ & $61(61.0)$ & \\
\hline IVa & $59(46.8)$ & $50(29.9)$ & & $38(38.0)$ & $39(39.0)$ & \\
\hline Smoking & & & 0.857 & & & 0.776 \\
\hline Yes & $53(42.1)$ & $72(36.5)$ & & $43(43.0)$ & $45(45.0)$ & \\
\hline No & $73(57.9)$ & $95(56.9)$ & & $57(57.0)$ & $55(55.0)$ & \\
\hline CCRT cycles & & & 0.983 & & & 0.171 \\
\hline$<2$ & $18(14.3)$ & $28(16.8)$ & & $12(12.0)$ & $19(19.0)$ & \\
\hline$\geq 2$ & $108(85.7)$ & $139(83.2)$ & & $88(88.0)$ & $81(81.0)$ & \\
\hline IC cycles & & & $<0.001$ & & & 0.637 \\
\hline$<2$ & $11(8.7)$ & 47 (28.1) & & $11(11.0)$ & $9(9.0)$ & \\
\hline$\geq 2$ & 115 (91.3) & $120(71.9)$ & & $89(89.0)$ & $91(91.0)$ & \\
\hline BMI $\left(\mathrm{kg} / \mathrm{m}^{2}\right)$ & & & 0.785 & & & 0.752 \\
\hline$<18$ & $5(4.0)$ & $8(4.8)$ & & $5(5.0)$ & $5(5.0)$ & \\
\hline $18-24$ & $78(61.9)$ & $108(64.7)$ & & $58(58.0)$ & $63(63.0)$ & \\
\hline$>24$ & $43(34.1)$ & $51(30.5)$ & & $37(37.0)$ & $32(32.0)$ & \\
\hline Histology & & & 0.751 & & & 0.359 \\
\hline I & $1(0.8)$ & $1(0.6)$ & & $0(0.0)$ & $1(1.0)$ & \\
\hline II & $1(0.8)$ & $3(1.8)$ & & $1(1.0)$ & $3(3.0)$ & \\
\hline III & $124(98.4)$ & 163 (97.6) & & $99(99.0)$ & $96(96.0)$ & \\
\hline
\end{tabular}

IC: induction chemotherapy; CCRT: concurrent chemoradiotherapy; TP: docetaxel and cisplatin; TPF: docetaxel, cisplatin and fluorouracil; DDP: cisplatin; BMI: body mass index.

Table 4. Multivariate analyses of prognostic factors in 200 patients with nasopharyngeal carcinoma after propensity score matching.

\begin{tabular}{llll}
\hline Endpoint & Variable & Hazard ratio & $95 \%$ CI \\
\hline OS & Clinical stage (III vs. IVa) & 2.646 & $(1.179-5.939)$ \\
& smoking (yes vs. no) & 2.310 & $(1.033-5.169)$ \\
PFS & Clinical stage (III vs. IVa) & 2.618 & $(1.302-5.264)$ \\
DMFS & Clinical stage (III vs. IVa) & 4.288 & $(1.529-12.02)$ \\
LRRFS & CCRT (<2 cycles vs. $\geq 2$ cycles) & 0.310 & 0.018 \\
\end{tabular}

CCRT: concurrent chemoradiotherapy; HR: hazard ratio. 
Table 5. Adverse events during IC after propensity score matching

\begin{tabular}{|c|c|c|c|c|c|c|c|c|c|c|}
\hline \multirow[t]{2}{*}{ Adverse events } & \multicolumn{2}{|c|}{ TP+DDP (case \%) } & \multicolumn{2}{|c|}{$\mathrm{TP}+\mathrm{TP}($ case $\%)$} & \multirow[t]{2}{*}{$P$} & \multicolumn{2}{|c|}{$\mathrm{TPF}+\mathrm{DDP}$ regimen (case \%) } & \multicolumn{2}{|c|}{$\mathrm{TP}+\mathrm{TP}$ regimen (case\%) } & \multirow[t]{2}{*}{$P$} \\
\hline & Grade 0-2 & Grade 3-4 & Grade $0-2$ & Grade 3-4 & & Grade $0-2$ & Grade 3-4 & Grade $0-2$ & Grade 3-4 & \\
\hline \multicolumn{11}{|l|}{ Hematologic } \\
\hline Leukocytopenia & $155(93.4)$ & $11(6.6)$ & $150(90.4)$ & $16(9.6)$ & 0.315 & $93(93.0)$ & $7(7.0)$ & $90(90.0)$ & $10(10.0)$ & 0.447 \\
\hline Neutropenia & $152(91.6)$ & $14(8.4)$ & $147(88.6)$ & $19(11.4)$ & 0.359 & $85(85.0)$ & $15(15.0)$ & $89(89.0)$ & $11(11.0)$ & 0.400 \\
\hline Anemia & $165(99.4)$ & $1(0.6)$ & $165(99.4)$ & $1(0.6)$ & 1.000 & $99(99.0)$ & $1(1.0)$ & $99(99.0)$ & $1(1.0)$ & 1.000 \\
\hline Thrombocytopenia & $165(99.4)$ & $1(0.6)$ & $166(100)$ & $0(0)$ & 1.000 & $99(99.0)$ & $1(1.0)$ & $100(100)$ & $0(0)$ & 1.000 \\
\hline \multicolumn{11}{|l|}{ Nonhematologic } \\
\hline Liver function & $166(100)$ & $0(0)$ & $166(100)$ & $0(0)$ & 1.000 & $100(100)$ & $0(0)$ & $100(100)$ & $0(0)$ & 1.000 \\
\hline Renal function & $166(100)$ & $0(0)$ & $166(100)$ & $0(0)$ & 1.000 & $100(100)$ & $0(0)$ & $100(100)$ & $0(0)$ & 1.000 \\
\hline Hypoalbuminemia & $166(100)$ & $0(0)$ & $166(100)$ & $0(0)$ & 1.000 & $100(100)$ & $0(0)$ & $100(100)$ & $0(0)$ & 1.000 \\
\hline Nausea/vomiting & $162(97.6)$ & $4(2.4)$ & $163(98.2)$ & $3(1.8)$ & 0.702 & $90(90.0)$ & $10(10.0)$ & $98(98.0)$ & $2(2.0)$ & 0.033 \\
\hline
\end{tabular}

IC: induction chemotherapy; TP: docetaxel and cisplatin; TPF: docetaxel, cisplatin and fluorouracil; DDP: cisplatin. Adverse events were graded according to the National Cancer Institute Common Toxicity Criteria, version 4.0.

Table 6. Adverse events during CCRT after propensity score-matched

\begin{tabular}{|c|c|c|c|c|c|c|c|c|c|c|}
\hline \multirow[t]{2}{*}{ Adverse event } & \multicolumn{2}{|c|}{ TP+DDP regimen (case \%) } & \multicolumn{2}{|c|}{$\mathrm{TP}+\mathrm{TP}$ regimen (case \%) } & \multirow[t]{2}{*}{$P$-value } & \multicolumn{2}{|c|}{ TPF+DDP regimen (case \%) } & \multicolumn{2}{|c|}{$\mathrm{TP}+\mathrm{TP}$ regimen (case\%) } & \multirow[t]{2}{*}{$P$-value } \\
\hline & Grade $0-2$ & Grade 3-4 & Grade $0-2$ & Grade 3-4 & & Grade $0-2$ & Grade 3-4 & Grade $0-2$ & Grade 3-4 & \\
\hline \multicolumn{11}{|l|}{ Hematologic } \\
\hline Leukocytopenia & $133(80.1)$ & $33(19.9)$ & $93(56.0)$ & $73(44.0)$ & $<0.001$ & $73(73.0)$ & $37(37.0)$ & $58(58.0)$ & $42(42.0)$ & 0.211 \\
\hline Neutropenia & $139(83.7)$ & $27(16.3)$ & $100(60.2)$ & $66(39.8)$ & $<0.001$ & $77(77.0)$ & $33(33.0)$ & $62(62.0)$ & $38(38.0)$ & 0.460 \\
\hline Anemia & $165(99.4)$ & $1(0.6)$ & $163(98.2)$ & $3(1.8)$ & 0.623 & $93(93.0)$ & $7(7.0)$ & $97(97.0)$ & $3(3.0)$ & 0.331 \\
\hline Thrombocytopenia & $165(99.4)$ & $1(0.6)$ & $165(99.4)$ & $1(0.6)$ & 1.000 & $95(95.0)$ & $5(5.0)$ & $99(99.0)$ & $1(1.0)$ & 0.212 \\
\hline \multicolumn{11}{|l|}{ Nonhematologic } \\
\hline Liver function & $162(97.6)$ & $4(2.4)$ & $164(98.8)$ & $2(1.2)$ & 0.685 & $100(100)$ & $0(0)$ & $100(100)$ & $0(0.0)$ & 1.000 \\
\hline Renal function & $166(100)$ & $0(0)$ & $166(100)$ & $0(0)$ & 1.000 & $100(100)$ & $0(0)$ & $100(100)$ & $0(0.0)$ & 1.000 \\
\hline Hypoalbuminemia & $166(100)$ & $0(0)$ & $166(100)$ & $0(0)$ & 1.000 & $100(100)$ & $0(0)$ & $100(100)$ & $0(0.0)$ & 1.000 \\
\hline Oral mucositis & $138(83.1)$ & $28(16.9)$ & $106(63.9)$ & $60(36.1)$ & $<0.001$ & $77(77.0)$ & $23(23.0)$ & $67(67.0)$ & $33(33.0)$ & 0.115 \\
\hline Nausea/vomiting & $161(97.0)$ & $5(3.0)$ & $151(91.0)$ & $15(9.0)$ & 0.035 & $82(82.0)$ & $18(18.0)$ & $92(92.0)$ & $8(8.0)$ & 0.036 \\
\hline
\end{tabular}

CCRT: concurrent chemoradiotherapy; TP: docetaxel and cisplatin; TPF: docetaxel, cisplatin and fluorouracil; DDP: cisplatin. Adverse events were graded according to the

National Cancer Institute Common Toxicity Criteria, version 4.0.

Docetaxel is used in the treatment of many solid tumors by blocking mitosis to achieve an antitumor effect[27]. A retrospective study demonstrated that docetaxel combined with radiotherapy had a similar clinical effect as DDP combined with radiotherapy in locoregionally advanced NPC[20]. Docetaxel showed great drug activity and an improved radiotherapy sensitization effect in head and neck cancers[27]. Therefore, whether the combination of DDP plus docetaxel concurrent with radiotherapy can further improve the survival rate of patients with locoregionally advanced NPC needs to be investigated. A phase II prospective study evaluated the short-term efficacy of TP-based IC followed by CCRT with TP regimen versus DDP alone in locoregionally advanced NPC and showed that the complete response (CR) rate of patients was similar in the two groups (93.3\% vs. $96.3 \%$, respectively)[28]. However, in our study, the long-term efficacy revealed that CCRT with a TP double-drug regimen could significantly improve patients' 5-year OS, PFS and DMFS compared with CCRT with DDP alone under the condition of the same IC regimen. Furthermore, the TP double-drug regimen was likely to improve OS by reducing the distant metastasis.

In recent years, the TPF + DDP regimen has become a category I recommendation for Epstein-Barr virus (EBV)-related NPC patients in the NCCN guidelines based on clinical trial results[12, 13]. The
TPF + DDP regimen had a high clinical value at the price of severe side effects, including hematologic toxicities and nonhematologic toxicities. Therefore, the optimal treatment regimen of locoregionally advanced NPC still needs further investigation. This study found that the 5-year OS, PFS, DMFS and LRRFS rates of the TP + TP group and the TPF + DDP group were not significantly different. However, the rate of grade $3 / 4$ nausea/vomiting in the $\mathrm{TP}+\mathrm{TP}$ group was lower than that in the TPF + DDP group during the IC and CCRT periods, which was the main clinical benefit in the TP + TP group.

Xie et al.[28] reported that grade 3/4 hematologic toxicities in the TP + TP group were higher than those in the TP + DDP group during CCRT, which was consistent with the results of our study. There was no significant difference in grade $3 / 4$ oral mucositis between the two groups in Xie's study[28], which showed $78.6 \%$ in the TP + TP group and $76.0 \%$ in the $\mathrm{TP}+\mathrm{DDP}$ group. However, our study demonstrated that patients in the TP + TP group had higher rates of oral mucositis than those in the TP + DDP group ( $36.0 \%$ vs. $16.9 \% ; P<0.001)$. The different results in this study and Xie's study may be related to the different radiotherapy techniques. In the era of IMRT, the rate of oral mucositis has significantly decreased[29]. The application of fluorouracil in IC and high-dose DDP $\left(100 \mathrm{mg} / \mathrm{m}^{2}\right)$ in CCRT may result in the higher rates of grade $3 / 4$ nausea/vomiting in 
the TPF + DDP group than in the TP + TP group.

At present, few studies have been published on the clinical value of CCRT with a double-drug regimen for locoregionally advanced NPC. This study confirmed the positive clinical value of CCRT with a TP double-drug regimen, which significantly decreased the rate of distant metastasis and improved the OS. The reason for this conclusion may be attributed to the fact that CCRT with a double-drug regimen was superior to CCRT with a single-drug regimen in preventing lymph node recurrence and distant metastasis. In this study, although the hematologic toxicities of the TP regimen were worse than those of the DDP alone regimen during CCRT, they were under clinical control. In summary, it is feasible to apply CCRT with a TP regimen in clinic. Furthermore, although the 5-year OS in the TP + TP group was similar to that in the TPF + DDP group, there was lower grade $3 / 4$ nausea/vomiting toxicity in the TP + TP group. Therefore, TP induction chemotherapy followed by CCRT with a TP regimen could be adopted for the treatment of locoregionally advanced NPC.

This study has some limitations. Because biases are inevitable in a retrospective study, we utilized PSM to exclude observable confounding factors. Furthermore, unknown potential confounding factors along with some lost cases are also issues. Additionally, some toxicities in some outpatients were not recorded adequately. Therefore, the results of this study need to be confirmed by a large prospective clinical study.

\section{Conclusion}

In patients with locoregionally advanced NPC treated with TP-based IC, compared with CCRT with DDP alone, the following CCRT with TP regimen decreased the rate of distant metastasis and improved OS; thus, we can draw the conclusion that CCRT with double-drug chemotherapy improved survival. Furthermore, we found that the 5-year OS, PFS, DMFS and LRRFS rates were similar between the TP + TP group and the TPF + DDP group, but the rates of acute adverse events of grade 3/4 nausea/vomiting responses were higher in the TPF + DDP group than in the TP + TP group during the IC and CCRT period; this was the main clinical benefit in the TP + TP group. Therefore, it might be optimal to treat patients with locoregionally advanced NPC with TP-based IC followed by CCRT with TP.

\section{Abbreviations}

NPC: nasopharyngeal carcinoma; IC: induction chemotherapy; IMRT: intensity-modulated radiotherapy; CCRT: concurrent chemoradiotherapy;
PSM: propensity score matching; EBV: Epstein-Barr virus; TP: docetaxel plus cisplatin; TPF: docetaxel, cisplatin and fluorouracil; DDP: cisplatin; NCI-CTCAE 4.0: National Cancer Institute Common Terminology Criteria for Adverse Events Version 4.0; GTV: gross tumor volume; CTV: clinical tumor volume; OS: overall survival; DMFS: distant metastasis-free survival; PFS: progression-free survival; LRRFS: locoregional relapse-free survival; HR: hazard ratio; BMI: body mass index; CI: confidence interval; WHO: World Health Organization; NCCN: National Comprehensive Cancer Network.

\section{Acknowledgements}

\section{Author Contributions}

Rong-Hui Zheng and Tai-Ze Yuan were guarantors of the entire study. Rong-Hui Zheng, Tai-Ze Yuan and Hao-Yun Tao developed the study concepts and designed this study. Ze-Jiang Zhan, Hao-Yun Tao, Wen-Ze Qiu and Kai Liao collected all the clinical data needed. Ze-Jiang Zhan and Hao-Yun Tao performed the statistical analyses. Ya-Wei Yuan, Tai-Ze Yuan, Ze-Jiang Zhan and Hao-Yun Tao prepared and edited the manuscript. All authors read and approved the final manuscript.

\section{Ethical approval}

This study was approved by the ethics committee of our hospital and has therefore been performed in accordance with the ethical standards described in the 1964 Declaration of Helsinki and its subsequent amendments (No. P2020-004).

\section{Consent for publication}

All authors have given their approval for publication of this manuscript.

\section{Competing Interests}

The authors have declared that no competing interest exists.

\section{References}

1. Chua MLK, Wee JTS, Hui EP, Chan ATC. Nasopharyngeal carcinoma. The Lancet. 2016; 387(10022):1012-1024.

2. Wee JT, Ha TC, Loong SL, Qian CN. Is nasopharyngeal cancer really a "Cantonese cancer"? Chin J Cancer. 2010; 29(5):517-526.

3. Afqir S, Ismaili N, Errihani H. Concurrent chemoradiotherapy in the management of advanced nasopharyngeal carcinoma: current status. J Cancer Res Ther. 2009; 5(1):3-7.

4. Tan WL, Tan EH, Lim DW, Ng QS, Tan DS, Jain A, et al. Advances in systemic treatment for nasopharyngeal carcinoma. Chin Clin Oncol. 2016; 5(2):21.

5. Tao CJ, Liu X, Tang LL, Mao YP, Chen L, Li WF, et al. Prognostic scoring system for locoregional control among the patients with nasopharyngeal carcinoma treated by intensity-modulated radiotherapy. Chin J Cancer. 2013; 32(9):494-501.

6. Al-Sarraf M, LeBlanc M, Giri PG, Fu KK, Cooper J, Vuong T, et al. Chemoradiotherapy versus radiotherapy in patients with advanced nasopharyngeal cancer: phase III randomized Intergroup study 0099. J Clin Oncol. 1998; 16(4):1310-1317. 
7. Lin JC, Jan JS, Hsu CY, Liang WM, Jiang RS, Wang WY. Phase III study of concurrent chemoradiotherapy versus radiotherapy alone for advanced nasopharyngeal carcinoma: positive effect on overall and progression-free survival. J Clin Oncol. 2003; 21(4):631-637.

8. Wu X, Huang PY, Peng PJ, Lu LX, Han F, Wu SX, et al. Long-term follow-up of a phase III study comparing radiotherapy with or without weekly oxaliplatin for locoregionally advanced nasopharyngeal carcinoma. Ann Oncol. 2013; 24(8):2131-2136

9. Chen QY, Wen YF, Guo L, Liu H, Huang PY, Mo HY, et al. Concurrent chemoradiotherapy vs radiotherapy alone in stage II nasopharyngeal carcinoma: phase III randomized trial. J Natl Cancer Inst. 2011; 103(23):1761-1770.

10. Chen $\mathrm{L}, \mathrm{Hu} \mathrm{CS}$, Chen $\mathrm{XZ}$, Hu GQ, Cheng ZB, Sun Y, et al. Adjuvant chemotherapy in patients with locoregionally advanced nasopharyngeal carcinoma: Long-term results of a phase 3 multicentre randomised controlled trial. Eur J Cancer. 2017; 75:150-158.

11. Qiu WZ, Huang PY, Shi JL, Xia HQ, Zhao C, Cao KJ. Neoadjuvant chemotherapy plus intensity-modulated radiotherapy versus concurrent chemoradiotherapy plus adjuvant chemotherapy for the treatment of locoregionally advanced nasopharyngeal carcinoma: a retrospective controlled study. Chin J Cancer. 2016; 35:2.

12. Sun Y, Li W-F, Chen N-Y, Zhang N, Hu G-Q, Xie F-Y, et al. Induction chemotherapy plus concurrent chemoradiotherapy versus concurrent chemoradiotherapy alone in locoregionally advanced nasopharyngeal carcinoma: a phase 3, multicentre, randomised controlled trial. The Lancet Oncology. 2016; 17(11):1509-1520.

13. Chen YP, Tang LL, Yang Q, Poh SS, Hui EP, Chan ATC, et al. Induction Chemotherapy plus Concurrent Chemoradiotherapy in Endemic Nasopharyngeal Carcinoma: Individual Patient Data Pooled Analysis of Four Randomized Trials. Clin Cancer Res. 2018; 24(8):1824-1833.

14. National Comprehensive Cancer Network: NCCN Clinical Practice Guide-lines in Oncology for Head and Neck Cancer, Version 1, 2018. http://www.nccn.org/index .asp.

15. Hui EP, Ma BB, Leung SF, King AD, Mo F, Kam MK, et al. Randomized phase II trial of concurrent cisplatin-radiotherapy with or without neoadjuvant docetaxel and cisplatin in advanced nasopharyngeal carcinoma. J Clin Oncol. 2009; 27(2):242-249.

16. Curran WJ, Jr, Paulus R, Langer CJ, Komaki R, Lee JS, Hauser $\mathrm{S}$, et al. Sequential vs. concurrent chemoradiation for stage III non-small cell lung cancer: randomized phase III trial RTOG 9410. J Natl Cancer Inst. 2011; 103(19):1452-1460.

17. Sol ES, Lee TS, Koh SB, Oh HK, Ye GW, Choi YS. Comparison of concurrent chemoradiotherapy with cisplatin plus 5-fluorouracil versus cisplatin plus paclitaxel in patients with locally advanced cervical carcinoma. J Gynecol Oncol. 2009; 20(1):28-34.

18. Parisi E, Genestreti G, Sarnelli A, Ghigi G, Arpa D, Burgio MA, et al. Accelerated hypofractionated radiotherapy plus chemotherapy for inoperable locally advanced non-small-cell lung cancer: final results of a prospective phase-II trial with a long-term follow-up. Radiat Oncol. 2019; 14(1):112.

19. Fu ZZ, Li K, Peng Y, Zheng Y, Cao LY, Zhang YJ. Efficacy and toxicity of different concurrent chemoradiotherapy regimens in the treatment of advanced cervical cancer: A network meta-analysis. Medicine (Baltimore). 2017; 96(2):e5853.

20. Liao JF, Zhang Q, Du XJ, Lan M, Liu S, Xia YF. Concurrent chemoradiotherapy with weekly docetaxel versus cisplatin in the treatment of locoregionally advanced nasopharyngeal carcinoma: a propensity score-matched analysis. Cancer Commun (Lond). 2019; 39(1):40.

21. Zhao C, Han F, Lu LX, Huang SM, Lin CG, Deng XW. Intensity modulated radiotherapy for local-regional advanced nasopharyngeal carcinoma. Ai Zheng. 2004; 23(11 Suppl):1532-1537.

22. National Cancer Institute-Common Toxicity Criteria Adverse Events Versions 4. http://evs.nci.nih.gov/ftp1/CTCAE/CTCAE 4.03 2010.

23. Austin PC. The performance of different propensity score methods for estimating marginal hazard ratios. Stat Med. 2013; 32(16):2837-2849.

24. Sun X, Su S, Chen C, Han F, Zhao C, Xiao W. Long-term outcomes of intensity-modulated radiotherapy for 868 patients with nasopharyngeal carcinoma: an analysis of survival and treatment toxicities. Radiother Oncol. 2014; 110(3):398-403.

25. Peng L, Liu JQ, Chen YP, Ma J. The next decade of clinical trials in locoregionally advanced nasopharyngeal carcinoma. Br J Radiol. 2019; 92(1102):20181031

26. Ke QH, Zhou SQ, Du W, Lei Y, Huang M, Luo F. Early efficacy of taxotere and cisplatin chemo-radiotherapy for advanced cervical cancer. Asian Pac J Cancer Prev. 2012; 13(2):617-619.

27. Dreyfuss AI, Clark JR, Norris CM, Rossi RM, Lucarini JW, Busse PM, et al. Docetaxel: an active drug for squamous cell carcinoma of the head and neck. J Clin Oncol. 1996; 14(5):1672-1678.

28. Xie. F-Y, Zou. G-R, Hu. W-H, Qi. S-N, Peng. M, Li. J-S. Induction chemotherapy with docetaxel plus cisplatin(TP regimen) followed by concurrent chemoradiotherapy with TP regimen versus cisplatin in treating locally advanced nasopharyngeal carcinoma. Cancer Communications. 2009; 28(3):279-85.

29. Orlandi E, Iacovelli NA, Rancati T, Cicchetti A, Bossi P, Pignoli E, et al. Multivariable model for predicting acute oral mucositis during combined
IMRT and chemotherapy for locally advanced nasopharyngeal cancer patients. Oral Oncol. 2018; 86:266-272. 